\title{
The effect of normobaric hypoxic exposure on coagulation as measured by thromboelastography
}

\section{AUTHORS:}

Jonny Coppel, Edward Gilbert-Kawai, Tom Bottomley, Joel Sugarman and Daniel Martin.

\section{ABSTRACT:}

\section{Introduction:}

The physiological impact of hypoxia on coagulation has significant importance in the clinical setting, but it is not yet fully understood. Various static methods exist to investigate the process of coagulation, however, thromboelastography (TEG) provides a dynamic assessment of clot formation that can be quantitatively assessed.

\section{Method:}

Twenty-five participants were exposed to normobaric hypoxia (12.5\% oxygen) for eight hours. Venous blood was taken from the participants directly pre- and post-hypoxic exposure, and coagulation was tested using TEG. Coagulation variables assessed included reaction time, split point, alpha angle, kinetics and maximum amplitude.

\section{Results:}

Time taken for clot initiation, (assessed using the split point and reaction time) was significantly reduced after eight hours of hypoxic exposure. The split point reduced from a mean of 5.20 to 4.23 minutes ( $\mathrm{p}=0.022)$, whilst the reaction time reduced from 6.09 to 4.94 minutes ( $p=0.004$ ). Maximum amplitude, alpha angle and kinetics did not change significantly after hypoxic exposure.

\section{Conclusion:}


The results demonstrate that subacute normobaric hypoxic exposure increases the tendency for whole blood to coagulate, as demonstrated by a reduced split and reaction time using TEG.

KEYWORDS: Hypoxia, thromboelastography, haemostasis

\section{ABBREVIATIONS:}

APTT $=$ activated partial thromboplastin time

$\mathrm{CAT}=$ calibrated automatic thrombogram

$\mathrm{CO}_{2}=$ carbon dioxide

COPD $=$ chronic obstructive pulmonary disease

Deg $=$ degrees

$\mathrm{FiO}_{2}=$ fraction of inspired oxygen

$\mathrm{Hb}=$ haemoglobin

HCT = haematocrit

INR = international normalized ratio

$\mathrm{K}$-time $=$ kinetics time

$\mathrm{MA}=$ maximum amplitude

$\mathrm{Ml}=$ millilitres

$\mathrm{Mm}=$ millimetres

$\mathrm{NO}=$ nitric oxide

OSA = obstructive sleep apnoea

$\mathrm{PT}=$ prothrombin time

$\mathrm{R}$-Time $=$ reaction time

ROTEM = rotational thromboelastometry

$\mathrm{SP}=$ split point

$\mathrm{TEG}=$ thromboelastography

$\mathrm{vWF}=$ von Willebrand's factor

$\alpha$ angle $=$ alpha angle 


\section{INTRODUCTION}

The effect of hypoxia on coagulation remains to be fully determined. Numerous studies have investigated this topic ${ }^{1-16}$, however, the findings differ markedly and have been conflicting, perhaps due to dissimilar techniques used to assess coagulation. Traditionally, in clinical settings, coagulation is assessed and reported by platelet count, pro-thrombin time (PT), activated partial thromboplastin time (APTT) and the derived international normalized ratio (INR) ${ }^{1}$. A frequently cited limitation of these traditional measurements, however, is that they do not fully account for the cellular component of coagulation process, such as the role performed by circulating platelets and red blood cells ${ }^{8}$.

Alternative methods which strive to measure the whole dynamic process of blood coagulation, include rotational thromboelastometry (RoTEM), thromboelastography (TEG), and calibrated automatic thrombogram (CAT) assays using fluoroscopy. ROTEM and TEG measure the viscosity of the blood sample as it clots, using either a tension wire (TEG), or a mirror and optical detector (ROTEM). Whilst TEG and ROTEM are based on a similar principle, their measurement outputs cannot be compared directly and some studies found that anticoagulation/bleeding management practice could differ depending on which method was used $17,18,19$. Both TEG and ROTEM have gained popularity clinically to aid the identification and correction of coagulopathy.

In addition to differences in the method used to assess coagulation, a second factor that may account for the diversity of reported results is length for which study participants are exposure to hypoxia. According to the definitions put forth by Martin et al. ${ }^{1}$, acute hypoxia is classified as up to six hours exposure, subacute hypoxia as between six hours and seven days, and sustained hypoxia between seven and 90 days. The precise mode of hypoxia also varies between studies; some studies create a hypoxic environment by altering the fraction of inspired oxygen $\left(\mathrm{FIO}_{2}\right)$ (normobaric normoxia) whilst others lower the barometric pressure (hypobaric hypoxia) such as is experienced at high altitude or in a hypobaric chamber. 
To date, several studies have found a hypercoagulable state exists after acute, subacute and early sustained hypoxia, with durations of exposure varying from between five minutes to eight days $s^{5,7,8,11}$. Proposed mechanisms for the hypercoagulable state include, increased thrombin generation ${ }^{4}$, increased fibrinolysis factors ${ }^{7}$, increased coagulation factors and also a cellular component, specifically platelet aggregation ${ }^{5}$. Other studies, however, have found either no statistical significance, or statistical but not clinical significance, between coagulation variables before and after hypoxic exposure ${ }^{4,13,14,15}$. Lastly, one TEG study ${ }^{1}$ demonstrated a hypocoagulable state. In this study the mean time for clot initiation, clot formation kinetics and fibrin polymerisation were significantly prolonged after 17 days at $5300 \mathrm{~m}$. These results are corroborated by a recent study $^{16}$ that used ROTEM to show significant delays in clot formation after durations towards the upper limit of subacute hypoxia (seven days).

Given these varied results, further research into the effect of hypoxia on coagulation is required in order to better delineate the effect of time spent exposed to hypoxia. We therefore developed a protocol to assess the effects of subacute normobaric hypoxic exposure on blood coagulation using TEG.

\section{METHOD}

\section{Participant selection}

Ethical approval for this study was obtained by the University College London Committee on the Ethics of Non-NHS Human Research. Participants were healthy volunteers aged 65 years or below, who were not of high altitude ancestry and had passed a medical screening questionnaire.

\section{Study Setting}

Participants were exposed to eight hours of normobaric hypoxia in a purpose built hypoxic enclosure measuring $350 \mathrm{~cm}$ (length) x $250 \mathrm{~cm}$ (width) x $245 \mathrm{~cm}$ (height). The hypoxic gas within it was produced by a SCU-270 hypoxicator 
(Hypoxico, New York, USA), and the enclosure was fitted with a TX-1100DRA zirconium cell oxygen monitoring system (PureAire, Illinois, USA), which provided stable oxygen readings independent of changes in temperature, barometric pressure, and humidity. The oxygen concentration was maintained at $12.5 \%$, equivalent to an altitude of approximately $4500 \mathrm{~m}$. The temperature was maintained at $22^{\circ} \mathrm{C}$ (ECO1206SQ inverter split air conditioning unit; Toshiba, Tokyo, Japan), and a carbon dioxide $\left(\mathrm{CO}_{2}\right)$ scrubber was used to maintain a normal $\mathrm{CO}_{2}$ concentration in the enclosure. Participants could exit the chamber briefly if required, during which time they used a personal breathing apparatus connected to a reservoir of air containing $12.5 \%$ oxygen.

\section{Oxygen saturations and heart rate}

The oxygen saturation and heart rate were measured using a pulse oximeter (Onyx®, Nonin, MN, USA) at baseline and then after every two hours of hypoxic exposure.

\section{Blood sample collection and TEG}

Blood samples were taken from participants prior to, and immediately after the eight-hour hypoxic exposure. Blood was collected by single venipuncture of an antecubital vein without stasis using a 21-gauge butterfly needle (BD Valu-Set ${ }^{\mathrm{TM}}$ ) and a polypropylene syringe (Terumo® UK Ltd, Egham, UK). Samples were taken from different veins on the two occasions. $5 \mathrm{ml}$ of blood was collected during each sampling; $1 \mathrm{ml}$ of blood was immediately transferred to a polypropylene reagent tube containing kaolin (Haemoscope $₫$, Niles, IL, USA). The tube was then inverted five times to ensure adequate mixing of blood and kaolin. An aliquot of $360 \mu \mathrm{l}$ of kaolin activated blood was then transferred using reverse pipetting technique into a cuvette and placed within the analysis well of the Haemoscope 5000 TEG analyser (Haemonectics®). Coagulation was assessed with the TEG software (version 4.2.2). Analysis was always started within one minute of initial venipuncture, and was terminated when the sample's maximum amplitude had been achieved. Recorded variables upon completion of the TEG assay were: split point (SP), reaction time (R-time), kinetics (K-time), alpha angle ( $\alpha$-angle), and maximum amplitude (MA). SP and 
R-time are a measure of time till clot initiation, $\mathrm{K}$-time and $\alpha$-angle are a measure of clot formation kinetics and fibrin polymerization (see Table 1 for full explanation of TEG variables). All the TEG measurements were performed by trained investigators with clinical experience in this technique.

Table 1 - Explanation of TEG Variables and Clinical Significance

\begin{tabular}{|c|c|c|c|}
\hline Variable & Test Description & Clinical Significance & Normal range** \\
\hline SP (Split Point) & $\begin{array}{l}\text { Time to initial clot formation, } \\
\text { determined by the earliest } \\
\text { resistance detected. }\end{array}$ & $\begin{array}{l}\text { Time to initiation of clot } \\
\text { formation. Reflecting the } \\
\text { presence of clotting factors } \\
\text { and clot inhibitors. }\end{array}$ & \\
\hline R-time (Reaction) & $\begin{array}{l}\text { Time taken from initiation of } \\
\text { the TEG until the amplitude } \\
\text { has reached } 2 \mathrm{~mm}(\mathrm{~min})\end{array}$ & $\begin{array}{l}\text { Time to initiation of clot } \\
\text { formation. Reflecting the } \\
\text { presence of clotting factors } \\
\text { and clot inhibitors. }\end{array}$ & $3-8 \mathrm{~min}$ \\
\hline K-time (Kinetics) & $\begin{array}{l}\text { Time for trace to separate } \\
\text { from } 2 \text { to } 20 \mathrm{~mm} \text { (min) }\end{array}$ & $\begin{array}{l}\text { A measure of clot formation } \\
\text { kinetics and fibrin } \\
\text { polymerization }\end{array}$ & $1-3 \mathrm{~min}$ \\
\hline$\alpha$-angle (Alpha) & $\begin{array}{l}\text { Angle measured from } \\
\text { baseline at the R-time to K- } \\
\text { time on the curve. Therefore } \\
\text { directly related to K-time } \\
\text { (degrees) }\end{array}$ & $\begin{array}{l}\text { A measure of clot formation } \\
\text { kinetics and fibrin } \\
\text { polymerization }\end{array}$ & $55-78^{\circ}$ \\
\hline MA (maximum amplitude) & $\begin{array}{l}\text { Maximum amplitude of the } \\
\text { clot }(\mathrm{mm})\end{array}$ & $\begin{array}{l}\text { Maximum clot strength. } \\
\text { Measure of the properties of } \\
\text { fibrin clot and activity of } \\
\text { platelets }\end{array}$ & $51-69 \mathrm{~mm}$ \\
\hline
\end{tabular}

${ }^{* *}$ normal values given for citrated whole blood prepared with kaolin

\section{Statistical analysis}

Data from the TEG analysis were normally distributed, as determined by a Shapiro-Wilk test, and thus reported as mean and standard deviation (SD) for the group. Accordingly, pre- and post-hypoxia measurements were compared using the paired $t$-tests. All statistical calculations were performed on SPSS version 22 (IBM, USA), and a p-value of $<0.05$ was taken to indicate statistical significance. A post hoc power calculation was performed using G*Power 3.1 
software (www.gpower.hhu.de).

\section{RESULTS}

Twenty-eight participants took part in this study, thirteen (46.4\%) of whom were female (see Table 2). The median age of participants was 25 years. None reported any significant co-morbidities prior to the study, nor were any taking any medications that would directly affect blood coagulation. Five patients did report to taking oral contraceptive medications. Three participants were unable to complete the hypoxic protocol and they were excluded from the final analysis.

Table 2 - Demographics of the Subjects Included in this study

\begin{tabular}{|l|l|}
\hline Demographics & Subjects \\
\hline Male/female (\%) & $54 / 46$ \\
\hline Median age (years) & 25 \\
\hline $\begin{array}{l}\text { On oral } \\
\text { contraceptives }\end{array}$ & 5 \\
\hline Total & 28 \\
\hline
\end{tabular}

Oxygen saturation dropped significantly from baseline after two hours of hypoxic exposure (mean (SD): baseline 98.0\% (1.1), two hours 82.7\% (3.6), (p value $<0.001$ ) but then did not significantly change after that (mean (SD): four hours $84.8 \%$ (3.5), six hours 84.8\% (3.3), eight hours 85.4\% (4.0)). Heart rate increased significantly during hypoxic exposure (Mean (SD): baseline $68.6 \mathrm{bpm}$ (9.7), two hours 69.4 (11.1), four hours 76.9 (11.7), six hours 75.2 (9.98), eight hours 73.7 (10.8) (p-value <0.001)).

The SP-time decreased after eight hours of hypoxic exposure (PRE mean (standard deviation $(\mathrm{SD}))=5.20 \mathrm{~min}$ [3.7-7.0], POST $4.23 \mathrm{~min}[2.33-6.13] ; \mathrm{p}=$ 0.022)) as did the R-time (PRE $6.09 \mathrm{~min}$ [4.5-7.68], POST $4.94 \mathrm{~min}$ [2.86- 7.02] 
$($ see Figure 1$) ;(p$ value $=0.004))$. There were no other differences between the two time points (see Table 3).

TABLE 3: Means and standard deviations of coagulation variables before and after hypoxia

\begin{tabular}{|llllll|} 
& SP $(\mathrm{min})$ & R-time $(\mathrm{min})$ & K-time $(\mathrm{min})$ & $\alpha$ angle $\left(^{\circ}\right)$ & MA $(\mathrm{mm})$ \\
\hline Before & $5.20(1.80)$ & $6.09(1.59)$ & $1.82(0.40)$ & $63.8(7.03)$ & $58.8(8.64)$ \\
\hline After & $4.23(1.90)^{*}$ & $4.94(2.08)^{*}$ & $1.85(0.77)$ & $64.9(7.35)$ & $59.1(7.86)$ \\
\hline
\end{tabular}

Table 2. Table of means (including standard deviations) of coagulation variables as measured by TEG, before and after eight-hour exposure to normobaric hypoxia of $12.5 \%$. * indicates a statistically significantly change from baseline $(p<0.05) . n=25$.

Post hoc power calculations for the t-test results for the $\mathrm{R}$ time showed a power of 0.83 (effect size $0.61, \alpha=0.05$ ). The overall results remained unchanged if the participants taking oral contraceptives were removed from the calculations.

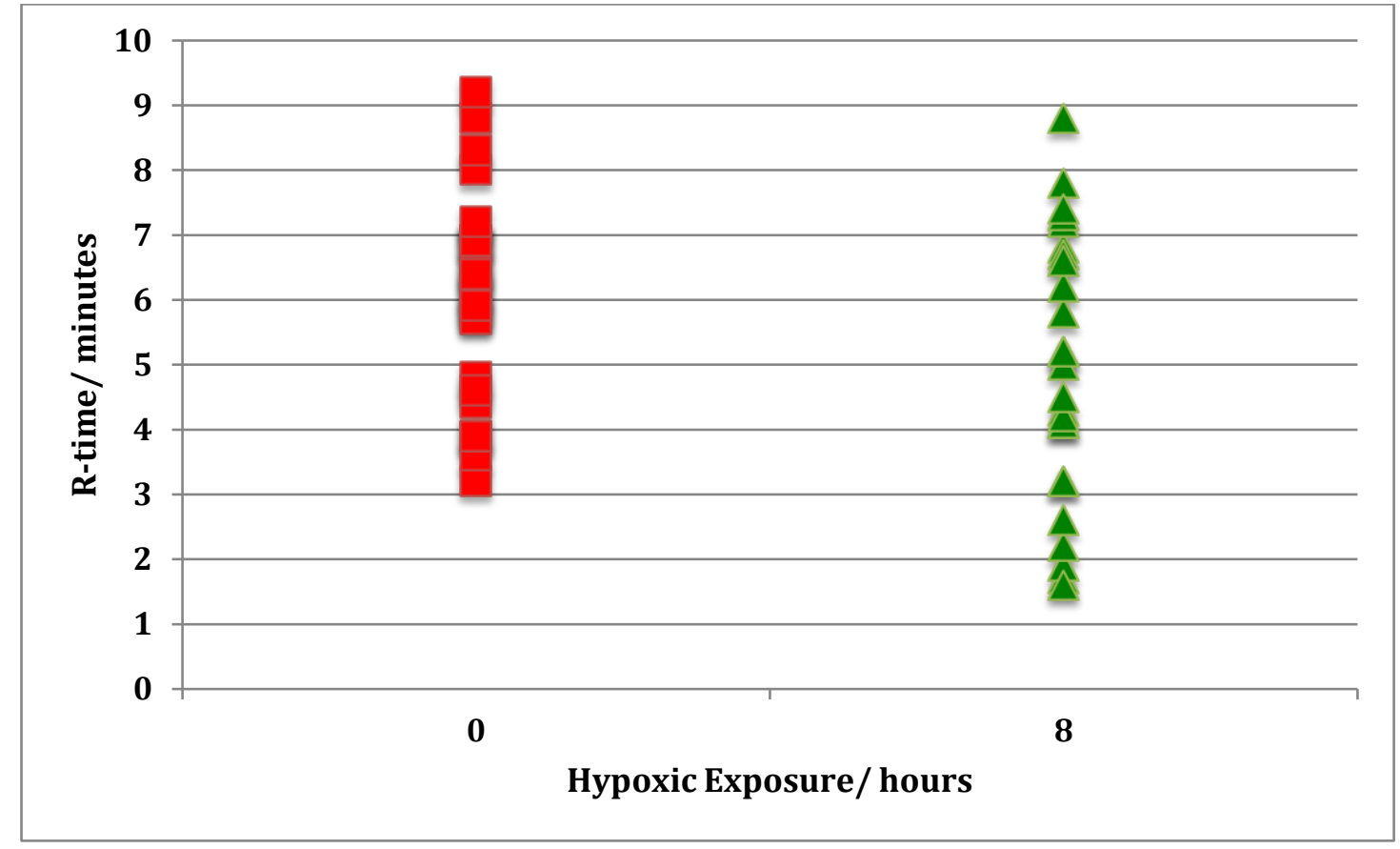

Figure 1: Dot plot to show the R-time of subject's blood samples before entering the hypoxic environment and after eight hours of exposure to $12.5 \% \mathrm{FiO}_{2}$. The R-time significantly decreased after eight hours of exposure. (mean (SD): PRE 6.09 min [4.5-7.68], POST 4.94 $\mathrm{min}$ [2.86- 7.02]; ( $\mathrm{p}$ value $=\mathbf{0 . 0 0 4})$ ). 


\section{DISCUSSION}

Given that there was a notable decrease in the mean SP and R time after eight hours of hypoxia, the predominant finding of this study is that clot initiation occurs sooner following subacute hypoxic exposure compared to baseline. It should be noted that although these changes were statistically significant, their clinical relevance may be small as the mean values after hypoxic exposure remained within the manufacturer's range of normal values. The lack of change in MA suggests no change in platelet function as a result of hypoxic exposure.

The results from this study are in agreement with most studies measuring coagulation, via traditional and more novel methods, in subacute hypoxia $5,7,8,9,11$. The changes in clot initiation demonstrated in this study may be caused by an acute stress response to hypoxia leading to activation of coagulation factors as demonstrated by Mannucchi et al. ${ }^{12}$ who found increased levels of plasminogen activator-inhibitor-1 activity and antigen, tissue plasminogen activator antigen, and prothrombin fragment 1 and 2 after subacute exposure to a similar oxygen concentration to this study. This response may be dose dependent given that Schobersberger et al's study not finding any differences in coagulation after ten hours of exposure to a lower hypoxic dose 21 .

Of the studies measuring whole blood coagulation over longer subacute hypoxic durations or prolonged hypoxia, the two studies using CAT5,7 (after six and eight days hypoxic exposure) found a hypercoagulable state, whereas three studies using the similar methods of ROTEM ${ }^{10,16}$ or TEG ${ }^{1}$ (after seven, nine and seventeen days hypoxic exposure) found significant delays in clot formation. This perhaps highlights the difficulties of comparing hypoxic exposures using different methodologies.

If we compare the results of this study to the studies of longer hypoxic durations using ROTEM or TEG, one possible explanation of this difference in results after different exposure durations, is that a biphasic pattern may exist. There may for example, be a biphasic response with initial shortening, and then lengthening of 
R-time, thus tending towards increased coagulation within hours, which then decreases after days. It is possible that after a certain period of exposure, the body's coagulation capacity is reduced, either through a deleterious effect of hypoxia whereby its regulation is impaired, or perhaps it is as a result of a physiological acclimatization to hypoxia. Park et al. ${ }^{22}$ have studied the effect of nitric oxide (NO) on coagulation. They found that it has an inhibitory effect on coagulation and suggest that it is mediated by its effect on platelet interaction with other coagulation factors in the plasma. Levett et al. ${ }^{23}$ subsequently demonstrated that sustained hypoxic exposure leads to an increase in NO activity and an increase in the concentration of NO metabolites, nitrite and nitrate, so perhaps a reduction in coagulation in sustained hypoxia compared to subacute may be a byproduct of the acclimatization process.

One possible factor leading to different results is the atmospheric pressure of the hypoxic exposure each study group was exposed to. The study in the Himalayas ${ }^{1}$ investigated the effect of hypobaric hypoxia, whereas this study used normobaric hypoxia. Whilst it is assumed that these two hypoxic environments have very similar physiological effects, it is still unclear whether this is in fact the case. Studies demonstrate differences in minute ventilation, NO levels and fluid retention ${ }^{21}$ thus dissimilarities in the coagulation pathways could also be a possibility. An additional difference between these two studies is exercise, in that trekking to $5300 \mathrm{~m}$ involves significant physical exertion, as well as temperature extremes and acclimatization - all of which could affect coagulation. Studies investigating the effects of exercise on coagulation have been so far inconclusive $25-27$.

Some studies, including this one, have found statistically significant changes in coagulation, yet the absolute values remain within normal clinical reference ranges. This does not necessarily negate the clinical relevance of the findings. Even if this level of change were clinically insignificant in a 'healthy' individual, in a patient whose baseline coagulation is already altered in conditions such as chronic obstructive pulmonary disease (COPD) and obstructive sleep apnoea 
(OSA), the addition of hypoxia to the hypercoagulable state may tip the patient significantly out of the normal range $28-31$.

\section{Future Research}

The altered coagulation demonstrated in this study, could be related to haematocrit. Westbury et al. ${ }^{2}$ studied whole blood coagulation in patients with congenital cyanotic heart disease, a patient population with chronically increased high haematocrit. Whilst one might have expected a prothrombotic state in this pathological condition, they actually found a hypocoagulable state. They demonstrated that this was a result of dilution of coagulation factors through increased red cell mass. On the other hand, high haematocrit caused by dehydration leads to increased thromboembolic events, for example in patients with diabetic ketoacidosis and hyperosmotic hyperglycaemic syndrome. An interesting area of further research would therefore be to investigate the independent effects of diuresis associated with hypoxia, and the increase in red cell mass secondary to hypoxia, on coagulation.

\section{Strengths and Limitations of the study}

This study provides a suitable comparison to the TEG results demonstrated from previous studies after sustained exposure to hypoxia ${ }^{1}$. The populations had a similar balance of genders. In this study, environmental conditions were controlled to reduce confounding factors such as the effect of hypothermia on coagulation. Finally, the use of TEG, rather than traditional clinical measurements of coagulation, gives a more comprehensive picture of the overall integrated coagulation process.

Limitations of the study include a failure to measure haemoglobin concentration or haematocrit in our participants. This would have given an indication of plasma concentration that may have changed as a result of hypoxia related diuresis. This might be relevant as changes in plasma concentration can affect coagulation $^{2}$. Whilst other studies have shown that haematocirt and haemoglobin concentrations do rise significantly in response to hypoxia after three days ${ }^{16}$, Schobersberger et al.'s study demonstrated that ten hours of 
hypoxic exposure, albeit a lower dose, did not change haemoglobin and packed cell volumes ${ }^{21}$. The $\mathrm{pH}$ of the participants' blood was also not measured and therefore any alteration in $\mathrm{pH}$ which may have independently affected coagulation could not be identified. Additionally, the level of hypoxic exposure in this investigation was not as high as that experienced at Everest Base Camp $(5300 \mathrm{~m}$, the equivalent hypoxic dose of approximately $10.5 \%$ ) in the paper by Martin et al. ${ }^{1}$ It is therefore possible, that a different pattern of coagulation is seen at oxygen concentrations lower than $12.5 \%$. There was no normoxic control group exposed to the conditions in this study. The participants spent the majority of the eight hours either sitting or lying down. It is possible that differences in coagulation could be as a result of this immobility similar to that proposed for the incidence of deep vein thrombosis on long haul flights ${ }^{21}$. Finally, there was inevitable selection bias in the sample population and most of the participants who volunteered were young and physically active individuals. This needs to be taken into account when extrapolating results to a clinical population.

\section{CONCLUSION}

Our results demonstrate that subacute hypoxic exposure increases whole blood coagulation as demonstrated by a reduced R-time on TEG. When considered in the context of other author's findings, this may suggest a time-dependent bimodal effect of hypoxia on coagulation. Given the importance of coagulation in managing patients with acute and chronic hypoxaemia , further work is required.

\section{COMPETING INTERESTS}

The Haemoscope 5000 TEG analyser was on loan from Haemonetics Corporation. 
Institute of Sport, Exercise and Health (London, UK) provided the laboratory for this experiment and we are grateful to Sarah Hayden for her help with the study. We would also like to thank all the participants for volunteering for the study and Haemonetics Corporation for the loan of the Haemoscope 5000 TEG analyser.

\section{REFERENCES}

1. Martin DS, Pate JS, Vercueil A, et al. Reduced coagulation at high altitude identified by thromboplasty. Blood Coagulation, Fibrinolysis and Cellullar Haemostasis 2012; 107:1066-1071.

2. Westbury SK, Lee $K$, Reilly-Stitt $C$, et al. High haematocrit in cyanotic congenital heart disease affects how fibrinogen activity is determined by rotational thromboelastometry. Thrombosis Research 2013; 132 e145151.

3. Redford DT, Paidy SR, Steinbremer EB, et al. Effects of profound hypoxemia on coagulation and fibrinolysis in normal individuals. Blood Coagulation and Fibrinolysis 2016; 27: 228-231.

4. Schaber $M$, Leichtfired $V$, Fries $D$, et al. Influence of acute normobaric hypoxia on haemostasis in volunteers with and without Acute Mountain Sickness. Biomedical Research International 2015; 1-9.

5. Ninivaggi $M$, De Laat $M$, Lance $M M$, et al. Hypoxia induces a prothrombotic state independently of the physical activity. PLOS ONE 2015; 1-14.

6. Birks JW, Klassen LW, Gurney CW, Hypoxia-induced thrombocytopaenia in mice. J lab. Clin. Med. 1975;86: 230-238.

7. Kicken $\mathrm{CH}$, Ninivaggi $\mathrm{M}$, Konings $\mathrm{J}$, et al. Hypobaric Hypoxia Causes Elevated Thrombin Generation Mediated by FVIII that is Balanced by Decreased Platelet Activation. Thromb Haemost. 2018; 118 (5): 883-892.

8. Bendz B, Rostrup M, Sevre K, et al. Association between acute hypobaric hypoxia and activation of coagulation in human beings. Lancet 2000;356(9242):1657-1658.

9. O'Brodovich HM, Andrew M, Gray GW, et al. Hypoxia alters blood coagulation istoring acute decompression in humans. J Appl Physiol 1984; 56: 666-670. iL is?

10. Modesti PA, Rapi S, Paniccia R, et al. Index measured at an intermediate altitude to predict impending acute mountain sick- ness. Med Sci Sports Exerc 2011;43(10):1811-1818. is șep:

11. Maher JT, Levine $P H$, and Cymerman A, et al. Human coagulation abnormalities during acute exposure to hypobaric hypoxia. Journal of 
Applied Physiology 1976; 41: 702- 707.

12. Mannucci PM, Gringeri A, Peyvandi F, et al. Short-term exposure to high altitude causes coagulation activation and inhibits fibrinolysis. Thrombosis and Haemostasis 2002; 87:342-343.

13. Toff WD, Jones CI, Ford I, et al. Effect of hypobaric hypoxia, simulating conditions during long-haul air travel, on coagulation, fibrinolysis, platelet function, and endothelial activation. JAMA 2006;295(19):2251-2261.

14. Crosby A, Talbot NP, Harrison $P$, et al. Relation between acute hypoxia and activation of coagulation in human beings. Lancet 2003;361(9376):22072208. istep:

15. Venemans-Jellema A, Schreijer AJM, Le Cessie S, et al. No effect of isolated long-term supine immobilization or profound prolonged hypoxia on blood coagulation. J Thromb Haemost 2014;12(06):902-909.

16. Rocke AS, Paterson GG, Barber MT, et al. Thromboelastometry and Platelet Function during Acclimatization to High Altitude. Thrombosis Haemostasis 2018: 118 (1):63-71.

17. Sankarankutty A, Nascimento B, Luz L, et al. TEG® and ROTEM® in trauma: similar test but different results? World Journal of Emergency Surgery 2012; 7 (Suppl 1): S3.

18. Coakley M, Reddy K, Mackie I. Transfusion Triggers in Orthotopic Liver Transplantation: A Comparison of the Thromboelastometry Analyzer, the Thromboelastogram, and Conventional Coagulation Tests. J CARDIOTHOR VASC AN 2006; 20 (4):548-553

19. Venema $L$, Post $W$, Hendriks $H$, et al. An assessment of clinical interchangeability of TEG and RoTEM thromboelastographic variables in cardiac surgical patients. Anesth Analg 2010; 111 (2) 339-344.

20. Hemler HC, Giesen $P$, Aldieri R, et al. The Calibrated Automated Thrombogram (CAT): a universal routine test for hyper- and hypocoagulability. Pathophysiol Haemost Thromb 2002; 32:249-253.

21. Schobersberger, W, Mittermayr, M, Fries, $D$, et al. Changes in blood coagulation of arm and leg veins during a simulated long-haul flight. Thromb Res 2007;119(3):293-300.

22. Park JW, Piknova B, Nghiem $K$, et al. Inhibitory effect of nitrite on coagulation processes demonstrated by thromboelastography. Nitric Oxide 2014; 40: 45-51.

23. Levett DZ, Fernandez BO, Riley HL, et al. The role of nitrogen oxides in human adaptations to hypoxia. Scientific Reports 2011; 1: 109-117.

24. Coppel J, Hennis P, Gilbert-Kawai E, et al. The physiological effects of hypobaric hypoxia versus normobaric hypoxia: a systemic review of crossover trials. Extreme Physiology \& Medicine 2015;4:2.

25. Bartsch P, Straub PW, Haeberli A, et al. Hypobaric hypoxia. The Lancet 2001: 357:955-956.

26. Van denburg PJ, Hospers JE, Mosterd WL, et al. Aging physical conditioning, and exercise-induced changes in haemostatic factors and reaction products. J Appl Physiol 2000; 88: 1558-1564. 
27. Gonzales JU, Thistlethwaite JR, Thompson BC, et al. Exercise-induced shear stress is associated with changes in plasma von Willebrand factor in older humans. Eur J Appl Physiol 2009; 106: 779-784.

28. Gegick S, Allen-Coore H, Bowling M. Chronic Obstructive Pulmonary disease: epidemiology, management, and impact on North Carolina. NCMJ 2013; 74: 411-414.

29. Piazza G, Goldhaber S, Kroll A, et al. Venous thromboembolism in patients with chronic obstructive pulmonary disease. Am J Med 2012; 125: 10101018.

30. Shitrit D, Peled N, Bar-Gil Shitrit A, et al. An association between oxygen desaturation and D-dimer in patients with obstructive sleep apnoea syndrome. Thromb Haemost 2005; 94: 1-4.

31. Wedzocha JA, Hurst JR. chronic obstructive pulmonary disease exacerbation and risk of pulmonary embolism. Thorax 2007; 62:103-104. 